\title{
Reaction kinetics of cassava starch graft anionic/nonionic-type polymer internal curing agents
}

\author{
Liu R J1,2,3* Sun $\mathrm{ZH}^{2}$, Xiang $\mathrm{WH}^{1}$, Chen $\mathrm{P}^{1,3}$, Zhou RZ ${ }^{1}$
}

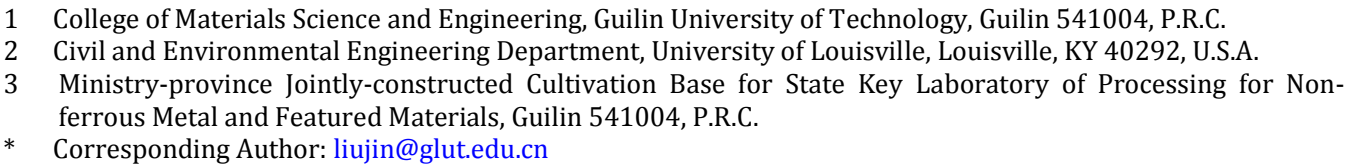

Received: 07-05-2018

Accepted: 29-08-2018

\begin{abstract}
Internal curing can help to improve the durability of concrete by preventing and minimizing initial cracks due to autogenous shrinkage and plastic shrinkage. Using a reliable internal curing agent is essential to the effectiveness of the internal curing process. This paper investigates the reaction kinetics of a starch graft anionic/nonionic-type polymer. The results demonstrate that initiator monomer concentration, and starch concentration are positively correlated with graft reaction rate $R_{p}$. Based on the research, the kinetics equation of this cassava starch graft anionic/nonionic-type polymer has also been developed, which coincides well with the law of free radical polymerization. The obtained $R_{p}$ equation is a firstorder dependence of the monomer concentration and the square root of the initiator concentration. And $R_{p}$ is further correlated to the reaction temperature based on a sigmoid function instead of a linear function. It is also found that the polymerization reaction is characterized by the coexisted disproportion termination and coupling termination.
\end{abstract}

Key words: free radical polymerization; graft reaction rate; kinetics; growth sigmoid curve; internal curing agent.

\section{Introduction}

Concrete, as the most commonly used material, creeps and shrinks during its service life. Concrete shrinkage, which causes volume change of the material, happens due to the loss of moisture (either internally or externally). It was found that the plastic shrinkage was the biggest reason for surface cracking of concrete pavements during the early age (Mindess et al., 2003). Early-age cracking is a predominant problem especially for high strength concrete/high performance concrete (HSC/HPC) due to the high autogenous shrinkage (self-desiccation) caused by its low water-to-cement ratio $(\mathrm{w} / \mathrm{c})$ and the usage of fine or ultra-fine mineral admixtures. These cracks would affect the durability and service life of concrete. Therefore, concrete curing plays a vital role in guaranteeing the development of the mechanical properties and service life performance of concrete.

In recent years, ideas of internal curing were proposed (Philleo, 1991; Weber \& Reinhardt, 1997; Bentz \& Snyder, 1999; Lura et al, 2014 ; Jensen \& Hansen, 2001; Jensen \& Hansen, 2002; Igarashi \& Watanabe, 2006). Internal curing is a process of providing concrete with additional water internally during the hydration process. This is done by using water absorbed in expanded shale, clay or slate (ESCS) lightweight aggregate(LWA)(Weber \& Reinhardt, 1997; Bentz \& Snyder, 1999; Lura et al, 2014 ) to partially replace the conventional aggregates in the mixture; or by using superabsorbent polymers (SAP) that would release water when needed during the selfdesiccation process (Jensen \& Hansen, 2001; Jensen \& Hansen, 2002; Igarashi \& Watanabe, 2006). By compensating the moisture loss due to self-desiccation, cracking caused by early-age shrinkage can be minimized and the ongoing hydration can also be improved. 
The effectiveness of internal curing is governed by the internal curing agents (ICA) or internal curing materials (ICM), such as LWA or SAP that used. SAP has been paid close attention to due to its high absorbency and its merit of swelling without dissolving in water. The present SAPs used in concrete are graft polymers made by starch, cellulose or the functional monomer such as acrylic acid, acrylonitrile, acrylamide (Jensen \& Hansen, 2001; Schröfl et al, 2012 ; Assmann \& Reinhardt, 2014). Controlling synthesis reaction is critical to acquire reliable ICAs. Especially for starch graft type polymer ICA, reaction kinetics strongly affects the stability of the reacting systems and the quality of the final ICAs. However, limited work has been done to report how to prepare stable and qualified starch graft type polymer ICA through reaction controlling. Lutfor $e t$ al. (2001) proposed several kinetics parameters and utilized ceric ammonium nitrate to investigate reaction kinetics of graft copolymerization of acrylonitrile and starch (Rahman et al, 2000). They also deduced the graft copolymerization reaction rate and derived the relation formula for monomer concentration and graft rate. Yu et al. (1999) studied the copolymerization kinetics of graft acrylonitrile onto starch by using benzoin ethyl ether as initiator, and derived the reaction rate equation and calculated the activation energy. They also deducted relative differential selective expression in the process of grafting under the experimental conditions. Wang (2003), explored the relationship among the concentration of initiator, monomer, starch graft, and the reaction rate by utilizing butyl acrylate, starch, ammonium per sulfate and emulsifier sodium dodecylsulfate. He proposed a graft reaction rate equation at the beginning of the reaction when the concentration of initiator was low. Similar research has been done by Yang et al. (2006) who obtained a simplified kinetics equation for two parameter-type universal graft polymerization reaction with experimental data. These previous works on reaction kinetics mainly confine to binary starch graft copolymerization reaction system without considering the effect of temperature. Recent research found that ternary starch graft system satisfies very well with the requirements of water absorbency, water retaining, and water releasing of ICAs because of its multiple functional groups (Liu, 2003).Therefore, knowing the reaction kinetics of the ternary graft system is useful to understand the relationship between the reaction systems and the quality of ICAs and is also helpful to control synthesis reaction. On the other side, temperature was also found to be a critical factor that closely related to molecular weight distribution, chain length, conversion of monomer, etc. (Lipsa et al, 2013). Therefore, temperature should also be included when modeling the reaction kinetics of a ternary starch graft system.

In this study, a kind of ICA called starch graft copolymer AM-AMPS (STAGAA) was developed. This ICA uses cassava starch, acrylamide (AM), and 2-acrylamide-2-methyl propyl sulfonic acid (AMPS) as the raw materials for the ternary system. Based on the preparation of the STAGAA, this paper attempts to further discuss how the related factors, such as concentration and temperature, affect the synthesis reaction. And the reaction rate equation for STAGAA ternary graft copolymer was also developed in this study. This would serve the purpose to predict synthesis reaction with eligible explanation.

\section{Experimental details}

\subsection{Raw Materials}

There are four kinds of reagents used in synthesis process, which are starch, monomer, initiator agent, and crosslinking agent. The moisture content of the cassava starch, the skeleton of STAGAA copolymer, was $11.2 \%$ and its side chain content was $83 \%$. The molecular structure of the cassava starch is illustrated in Figure 1. The amount of side chains plotted in Figure 1a represents the amount of active graft sites. The higher content of the side chain would lead to more active chemical reaction with the monomer (Moad \& Solomon, 2006).The C-C bond between C2 and C3 sites in the glucose ring is easy to break and then the hydroxide on C2 and C3 is easy to react (Tong \& Zhang, 2005; Zhang, 2001), as showed in Figure 1b. 


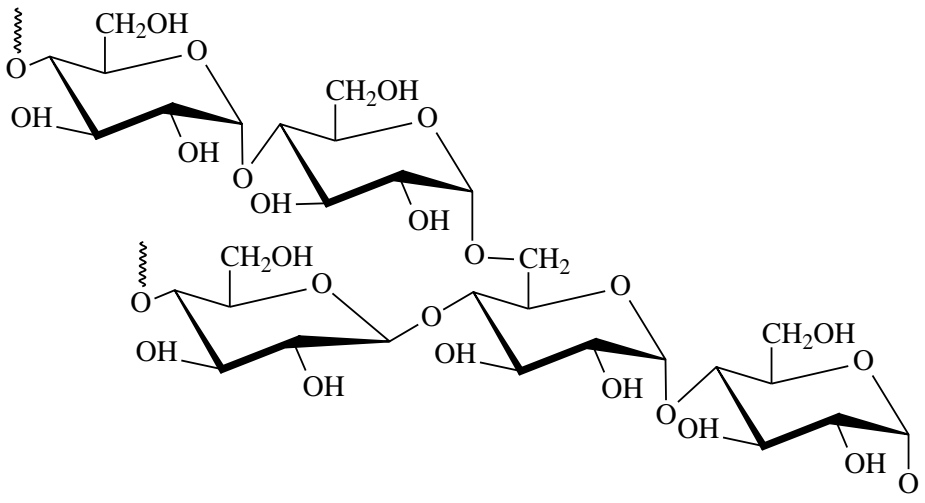

(a) Structure unit

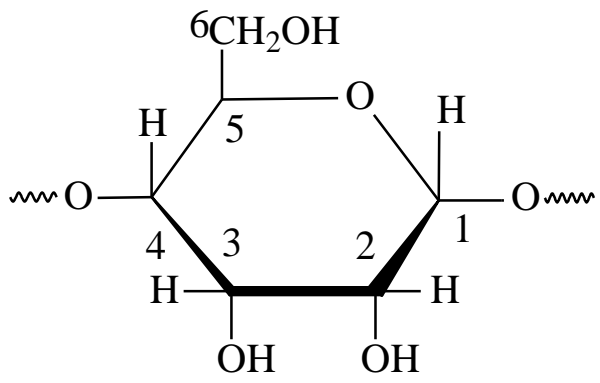

(b) Glucose ring

Fig 1. Molecular structure of amylopectin cassava starch

The used reagent monomers in STAGAA synthesis process were of two kinds: acrylamide (AM) that provides with nonionic amide group and 2-acrylamido-2-methylpropane sulfonic acid (AMPS) that provides with anionic sulfonic group. Ammonium persulfate (APS) and N,N'methylenebisacrylamide (NMBA) were used as an initiator agent and a crosslinking agent, respectively.

\subsection{Preparation of STAGAA}

The STAGAA copolymer was synthesized by aqueous solution method shown in Figure 2.

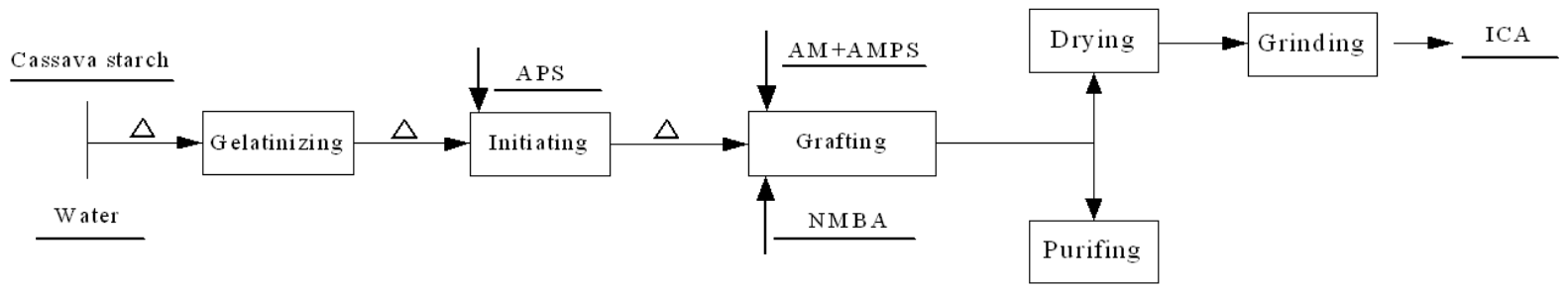

Fig 2. Flowchart of STAGAA synthesis.

First, the cassava starch was mixed and dissolved with distilled water, and then heated to 70 80 ${ }^{\circ} \mathrm{C}$ to gelatinize in a four-necked flask for about 30 minutes until the color turns colorless from white. Then, the action temperature was lowered to $60 \sim 70{ }^{\circ} \mathrm{C}$ and APS was added for initiating for 30 minutes. Next, AM, AMPS and NMBA were all dissolved in distilled water, and then dropped very slowly, and grafted and copolymerized for about 2 hours until a transparent or translucent gel-like substance was achieved. Finally, the gel-like product was precipitated in alcohol, then filtered and washed with distilled water repeatedly, and dried at $105^{\circ} \mathrm{Cin}$ a vacuum 
oven to constant weight, then crushed and ground to the required fineness to obtain the STAGAA powder. For the characterization purpose, part of the gel-like crude product was also put in a Soxhlet extractor with isopropanoltoreflux and extract for 24 hours. The purified sample was subsequently dried to a constant weight to remove unreacted monomers.

\subsection{Determination of Graft Reaction Rate}

In this study, the weight method was used to determinate the graft reaction rate, $R_{p}$. Firstly, a certain amount of the purified STAGAA copolymer was put into concentrated hydrochloric acid by refluxing to fully eliminate the starch backbone. Then, $1 \mathrm{~mol} / \mathrm{L}$ sodium hydroxide solution was used to neutralize the residual hydrochloric acid and $\mathrm{AgNO}_{3}$ solution was used to be hydrochloric acid until the $\mathrm{Cl}$ - is completely reacted. After that, the insoluble product was dried in a vacuum and its weight equaled the weight of monomer on the grafted side chain. The graft reaction rate, $R_{p}$, can be calculated according to the following equation based on the side chain weight.

$R_{p}(\mathrm{~mol} / \mathrm{L} \cdot \mathrm{min})=\frac{\text { grafted monomer weight onto side chain }}{\text { monomer molar mass } \times \text { reaction time } \times \text { reaction volume }}$

During the grafting reaction, several factors such as initiator concentration, monomer concentration, and starch concentration should be considered comprehensively, and $R_{p}$ can also be written as Eq (2):

$R_{p}=K \times[A]^{a}[B]^{b}[C]^{c}$

where $K$ represents the rate coefficient; $[A]$ is the initiator concentration (in mol/L); $[B]$ is the monomer concentration (in mol/L); $[C]$ is the starch concentration (in mol/L); $a, b$, and $c$ are reaction orders, respectively.

The logarithmic expression of the above mentioned Eq (2) can be shown in the following equation:

$\ln R_{p}=\ln K+a \ln [A]+b \ln [B]+c \ln [C]$

According to the experimental data, there is a liner correlation between $\ln R_{p}$ and $\ln [A]$, and the slope equals the coefficient $a$. Similarly, when plotting the curve of $\ln R_{p}$ versus $\ln [B]$ and $\ln [C]$ respectively, coefficient $b$ and $c$ can be obtained. Given a certain temperature, reaction rate $R_{p}$, reactants concentration $[A],[B]$ and $[C]$, reaction stages $a, b, c$, and the rate constant $K$ can be obtained from Eq (3).

\section{Results and discussion}

\subsection{IR analysis of STAGAA}

In Figure 3a, the absorption peak $3394.1 \mathrm{~cm}^{-1}$ presents the stretching vibration of alcohol - OH group in cassava starch, while $2931.3 \mathrm{~cm}^{-1}$ marks saturated C - H stretching vibration. Peaks of $1421.3 \mathrm{~cm}^{-1}, 1016 \mathrm{~cm}^{-1}$, and $1157 \mathrm{~cm}^{-1}$ represent asymmetric stretching vibration of C - O - C key symmetric in the glucose unit. In Figure $3 \mathrm{~b}, 3411.5 \mathrm{~cm}^{-1}$ shows that product retains the alcoholic alcohol - OH group absorption peak but blueshifts; New "shoulder peak" $3208.8 \mathrm{~cm}^{-1}$ addresses the stretching vibration of imino group (=NH) in AMPS; For $1670.1 \mathrm{~cm}^{-1}$ and $1043.3 \mathrm{~cm}^{-1}$, they are stretching vibration peak of carbonyl group $\mathrm{C}=\mathrm{O}$ and $\mathrm{S}=\mathrm{O}$ sulfonic group respectively. The above three characteristics are a clear indication that the monomer AM and monomer AMPS were grafted onto the cassava starch and formed the anionic and nonionic polymer STAGAA. 

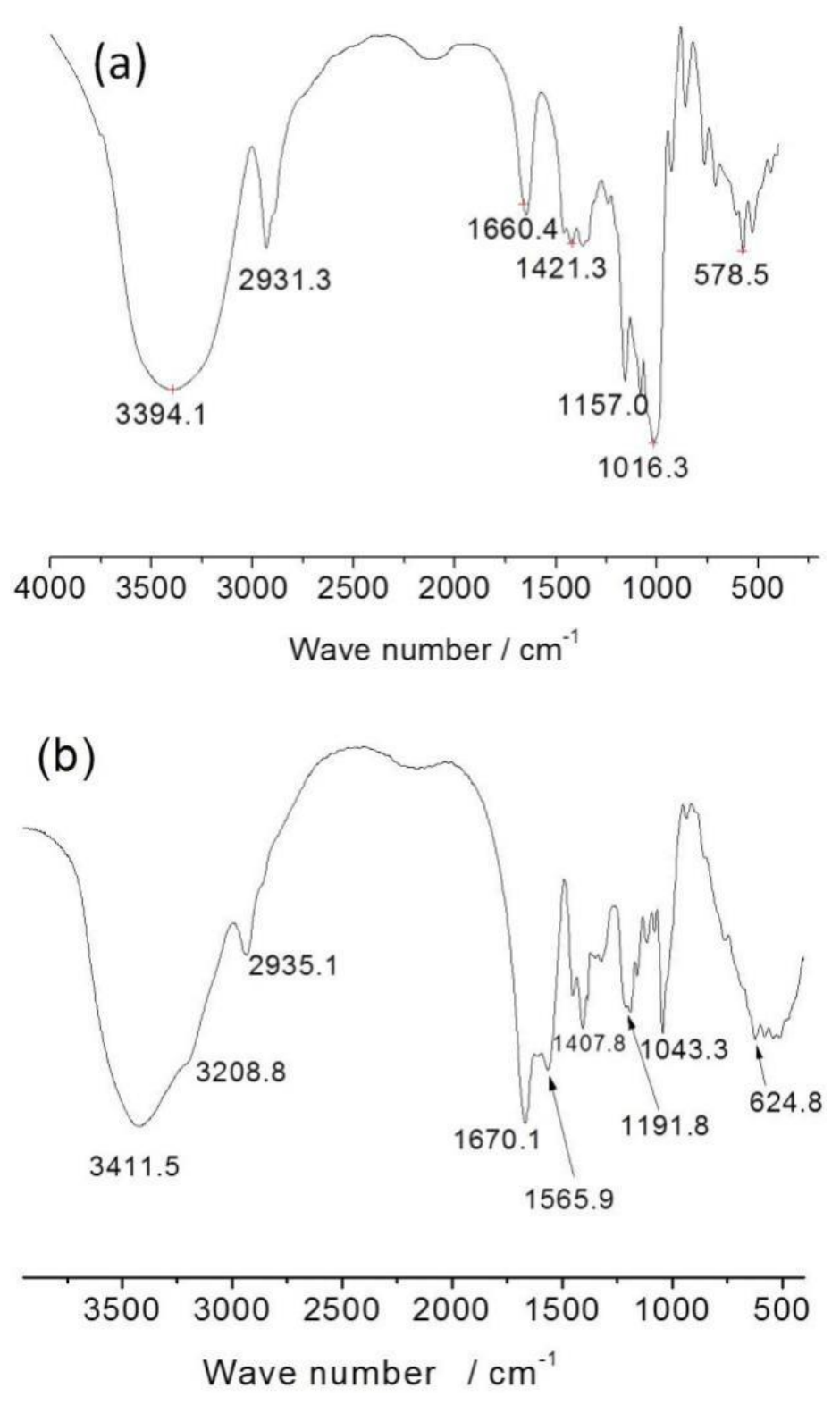

Fig 3. IR spectrograms of cassavastarch (a) andSTAGAA (b)

\subsection{SEM analysis of STAGAA}

Seen from Figure 4a, cassava starch particles have spherical or hemispherical in shape and smooth surface, and its size varies widely. In Figure 4b, STAGAA obviously characterizes by wrinkled surface, and its assemble as a layered structure. Its microstructure is clearly different from three-dimensional network of the common crosslinked SAP (Ren et al, 2012). According to the synthesis process and the surface morphology, the authors tentatively thought that the shape and its assemble state were formed as follows: the water-absorbed STAGAAs firstly lost internal water under the interaction with the environment, then the flexible strip or membrane STAGAAs gradually stacked, finally assembled as a special appearance of wrinkles. This kind of wrinkled surfaces and layered structure provides STAGAAs with a channel to absorb water and a lot of space to store water. 

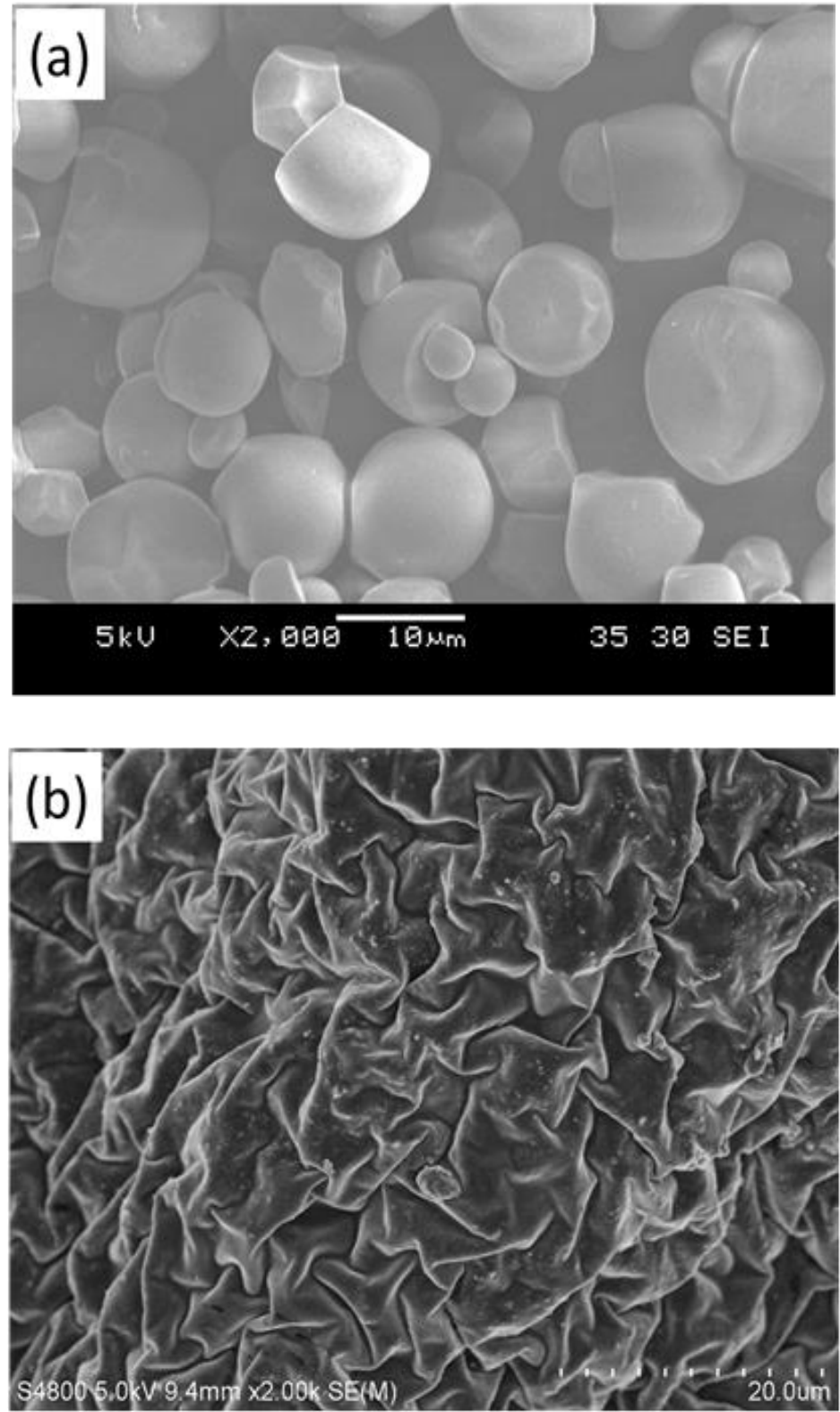

Fig 4. Morphology of cassava starch (a) and STAGAA (b).

\subsection{Determination of Early Reaction Time}

The monomer weight change grafted onto starch backbone has been recorded shown in Figure 5. It shows that the mean monomer mass grafted onto starch backbone increases linearly with the increasing reaction time during the early 30 minutes, but it increases slowly after that. This indicates that the reaction rate is approximately constant in early reaction time (30 minutes) and it can be considered as the initial reaction rate. 


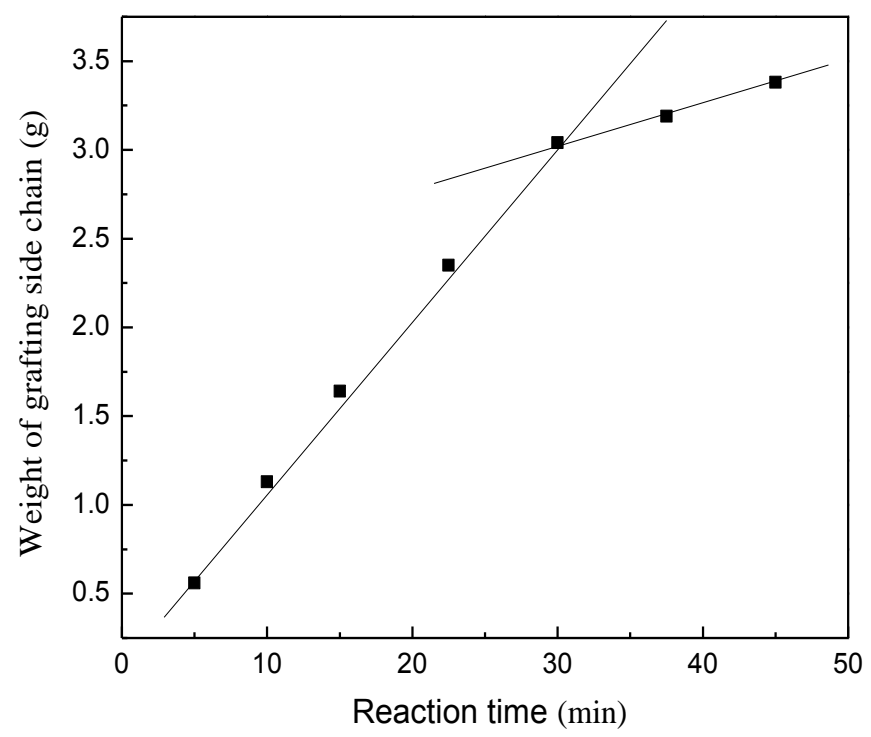

Fig 5. Curve of the grafted monomers weight changes with the reaction time

\subsection{Initiator Concentration}

In the study, starch concentration $[C]$ and monomer concentration $[B]$ were first kept constant at $0.23 \mathrm{~mol} /$ Land $1.5 \mathrm{~mol} / \mathrm{L}$. The material was reacted at $70{ }^{\circ} \mathrm{C}$ for 30 minutes. The initiator concentration $[A]$ was ranged from $0.01 \mathrm{~mol} / \mathrm{L}$ to $0.012 \mathrm{~mol} / \mathrm{L}$. The fitting curve of $\ln R_{p}$ is plotted in Figure 6. It shows that the curve $\ln R_{p}$ presents a positive linear correlation with $\ln [A]$ at early stage of the graft reaction. The linear correlation coefficient $R^{2}$ was 0.998 using the least squares linear regression fitting. Therefore, for the initiator, the reaction order (coefficient $a$ in Eq 3) was 0.509 .

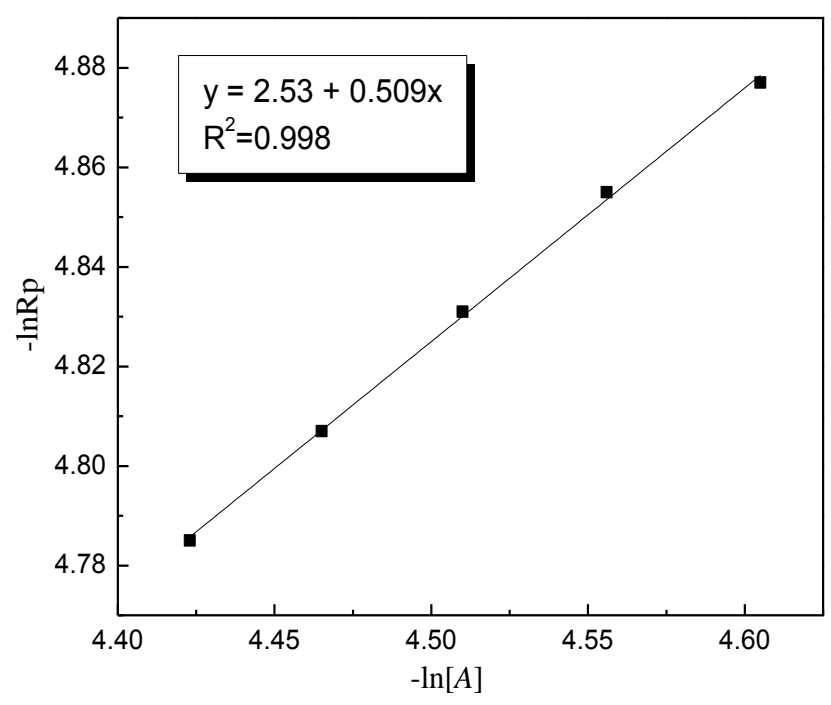

Fig 6. The relationship between $-\ln R_{p}$ and $-\ln [A]$

It should be noted that when the initiator concentration increases, starch free radicals and monomer free radicals increase accordingly. Thus, the number of graft reaction active center 
increases, which accelerates the synthesis reaction. The chain termination process of the alkene monomers is controlled by the diffusion process (Gugliemelli et al, 1972). When $R_{p}$ is proportional to the square root of the initiator concentration, it indicates that all the coupling reactions are terminated. However, it is difficult to realize the termination of all the coupling reactions because some free radicals are encased during the gel reaction process. In general, termination by disproportion and coupling coexist, and the graft reaction order of the initiator concentration ranges from 0.5 to 1.0 ( 0.5 for coupling termination, 1.0 for disproportionation termination). In this study, when the reaction order of initiator (coefficient $a$ ) goes up to 0.509 , it means that both the disproportion termination and the coupling termination coexist and the latter takes a dominant position.

\subsection{Monomer Concentration}

To find out the coefficient $b$ in Eq (3), the starch concentration $[C]$ and the initiator concentration $[A]$ were kept constant at $0.23 \mathrm{~mol} / \mathrm{L}$ and $0.012 \mathrm{~mol} / \mathrm{L}$, respectively, and the material was reacted at $70{ }^{\circ} \mathrm{C}$ for 30 minutes. The monomer concentration $[B]$ varied from 0.7 $\mathrm{mol} / \mathrm{L}$ to $1.5 \mathrm{~mol} / \mathrm{L}$ and the fitting curve of $R_{p}$ is presented in Figure 7.

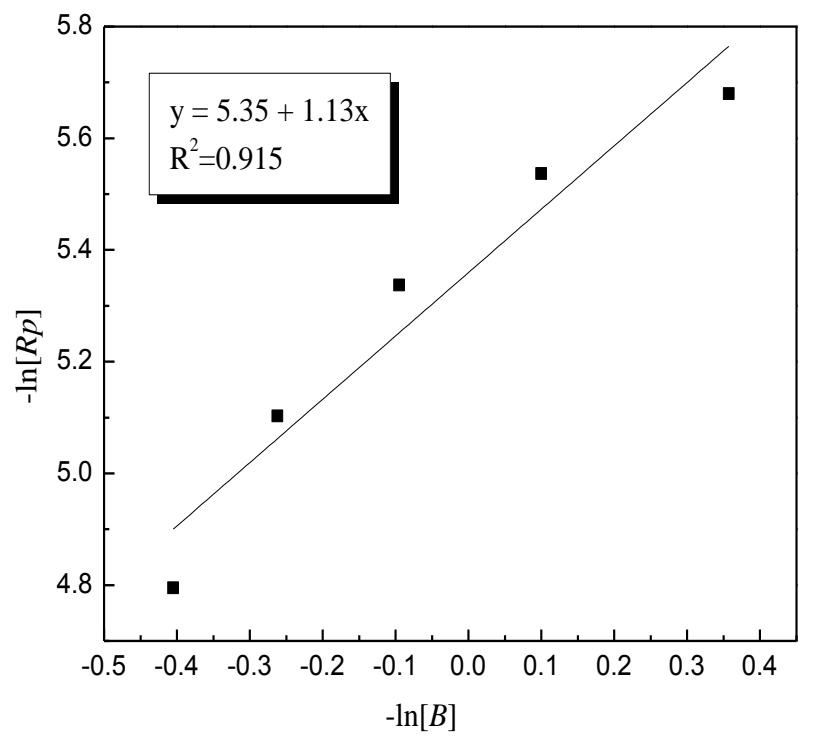

Fig 7. The relationship between $-\ln R p$ and $-\ln [\mathrm{B}]$

\subsection{Starch Concentration}

It can be seen that the curve of $R_{p}$ with $[B]$ exhibits a positive linear correlation within the range that studied. The $R^{2}$ is 0.915 and the reaction order (coefficient $b$ in Eq 3) is 1.135 for the monomer. This relationship indicates that low monomer concentration means monomer molecules have enough chance to cover the surface of the starch, so the active reaction center on the surface is relatively poor. Within a certain range, the monomer amount caused by free radicals increases, thus $R_{p}$ increases significantly.

In order to further study the relationship between $R_{p}$ and starch concentration $[C]$, the monomer concentration $[B]$ and the initiator concentration $[A]$ were kept at $1.5 \mathrm{~mol} / \mathrm{L}$ and $0.012 \mathrm{~mol} / \mathrm{L}$ with the graft reaction at $70{ }^{\circ} \mathrm{C}$ for 30 minutes. The chosen starch concentration was changed from $0.03 \mathrm{~mol} / \mathrm{L}$ to $0.23 \mathrm{~mol} / \mathrm{L}$. The correlation curve is shown in Figure 8. It shows that the $R_{p}$ presents a good linear relationship with the starch concentration. With the $R^{2}$ value of 0.999 , the obtained reaction order is 0.506 (coefficient $c$ in Eq 3) for the starch. 


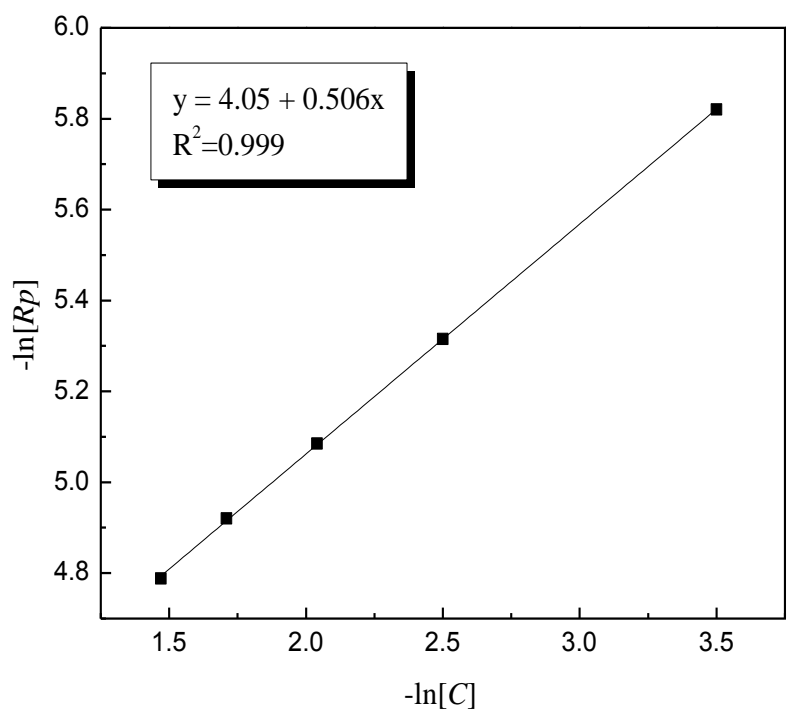

Fig 8. The correlation between $-\ln R p$ and $-\ln [\mathrm{C}]$

This linear correlation is due to the fact that the reaction occurs mainly on the surface of starch molecules at first. Then, with the increasing starch concentration, the active reaction sites on the surface of starch molecules will increase, which causes the significant increasing of $R_{p}$.

From the reaction orders of initiator concentration $[A]$, monomer concentration $[B]$, and starch concentration $[C]$, the $\mathrm{Eq}(2)$ of $R_{p}$ can be revised as follows:

$R_{P}=K \times[A]^{0.509}[B]^{1.135}[C]^{0.506}$

\subsection{Reaction Temperature}

In order to investigate the influencing factors on the rate constant $K$, both the $R_{p}$ and $K$ values are listed in Table I when the reaction temperature $T$ was changed from $50^{\circ} \mathrm{C}$ to $80^{\circ} \mathrm{C}$ while the concentration of starch, monomer, and initiator constant were kept at $0.23 \mathrm{~mol} / \mathrm{L}, 1.5 \mathrm{~mol} / \mathrm{L}$, and $0.012 \mathrm{~mol} / \mathrm{L}$, respectively.

Table 1. The Relationship of the Rp and $K$ with $T$

\begin{tabular}{|c|c|c|c|c|c|c|c|c|}
\hline \multirow{2}{*}{$\mathrm{T}$} & $\left({ }^{\circ} \mathrm{C}\right)$ & 50 & 55 & 60 & 65 & 70 & 75 & 80 \\
\cline { 2 - 8 } & $(\mathrm{K})$ & 323.15 & 328.15 & 333.15 & 338.15 & 343.15 & 348.15 & 353.15 \\
\hline \hline \multicolumn{2}{|c|}{$R_{p} \times 10^{3}$} & 1.079 & 1.576 & 2.988 & 5.01 & 7.905 & 8.295 & 8.412 \\
\hline \multicolumn{2}{|c}{$K\left(\mathrm{~min}^{-1}\right)$} & 0.0136 & 0.0199 & 0.0377 & 0.0631 & 0.0997 & 0.1046 & 0.1061 \\
\hline
\end{tabular}

The table indicates that both $R_{p}$ and $K$ increase with the increasing reaction temperature. However, the $R_{p}$ value increases slowly when temperature increases from 50 to $55^{\circ} \mathrm{C}$, it increases notably faster after that. This is due to the faster initiator $\left(\mathrm{NH}_{4}\right)_{2} \mathrm{~S}_{2} \mathrm{O}_{8}$ decomposition rate caused by the higher temperature. When the initiator produces more free radicals, the increased grafted monomer leads to the higher number of free radicals, causing graft reaction chain initiation and chain growth rate to increase correspondingly. At the same time, the higher temperature is beneficial to the starch gelatinization and the increase of active sites on the starch molecule surface, which makes the $R_{p}$ to increase in the end. 
According to Table 1, the relationship between the rate constant $K$ and the temperature $T$ (in Kelvin) is plotted in Figure 9. Instead of a linear relationship proposed by Lutfor et al. (2001) and Wang (2003), it can be seen from the figure that the $K$ - $T$ relationship satisfies a sigmoid function. The rate constant, which should be called rate coefficient, increases slightly within the low and high temperature ranges, but it increases sharply during intermediate temperature section. This indicates that the copolymer reaction of starch-graft-AM-AMPS is more complex than ordinary radical copolymerization. Since a ternary starch graft system is easier to form gel for copolymerization, the reaction temperature of STAGAA should not be limited to the range from 60 to $70^{\circ} \mathrm{C}$

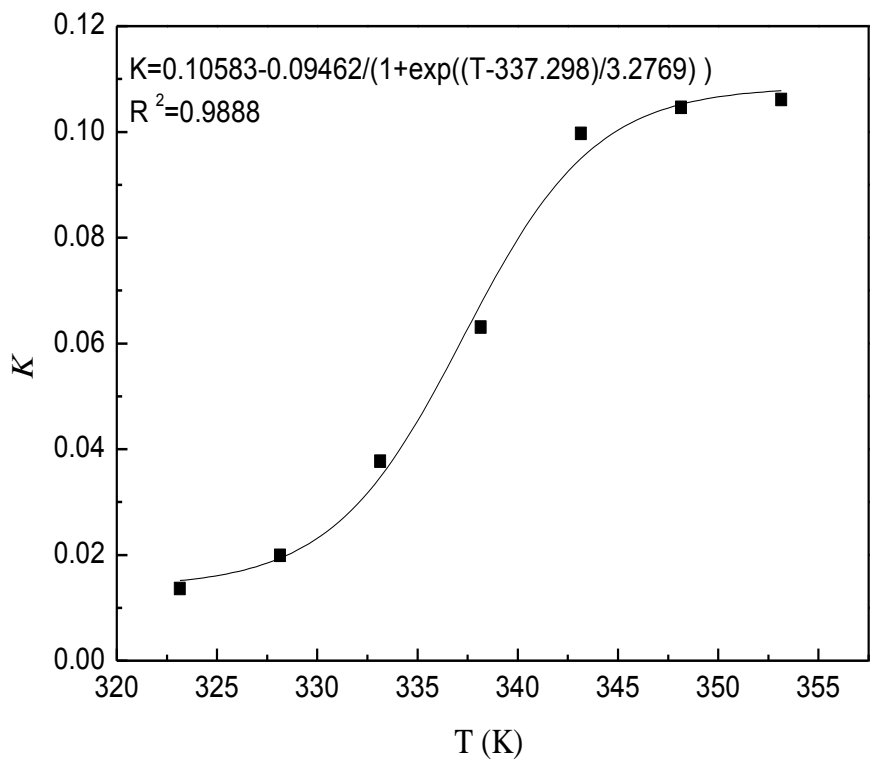

Fig 9. The fitting curve of $K$ with $T$

This relationship can also be expressed by Eq (5), where the correlation coefficient $R^{2}$ is 0.9888 .

$$
K=0.10853-0.09462 /(1+\exp ((T-337.289) / 3.2769))
$$

The final expression for $R_{p}(\mathrm{Eq} 4)$ can be rewritten as follows:

$$
R_{P}=(0.10853-0.09462 /(1+\exp ((T-337.289) / 3.2769))) \times[A]^{0.509}[B]^{1.135}[C]^{0.506}
$$

As Eq (6) includes the effect of temperature on starch graft reaction, it provides the production of such a starch graft copolymer with a solid background of the influence of processing condition on material reactivity in the future. This result is supported by the experimental data and can be used to verify the reaction kinetic related to the draft fractions of AM and AMPS on cassava starch.

\section{Conclusion}

Based on the represented research, following conclusions can be drawn. First, the developed reaction kinetics model for starch-graft-AM-AMPS copolymer can be expressed as:

$$
R_{P}=(0.10853-0.09462 /(1+\exp ((T-337.289) / 3.2769))) \times[A]^{0.509}[B]^{1.135}[C]^{0.506}
$$

Which agrees well with the law of free radical polymerization. The factors of initiator concentration $[A]$, monomer concentration $[B]$, starch concentration $[C]$ are positively correlated 
with $R_{p}$. When the correlation between temperature and $R_{p}$ is considered specifically, it can be expressed with a sigmoid function instead of a linear function. The research also found that the synthesis reaction of STAGAA copolymer is characterized by disproportion termination and coupling termination. Both terminations coexist and the latter is the dominant reaction format.

\section{Acknowledgements}

This research was financially supported by the National Natural Science Foundation of China (51202039, 21566008), National Science Foundation of Guangxi (2014GXNSFAA118314) and Science and technology development plan of Guangxi (1348011-2). The support from the Civil and Environmental Engineering Department at the University of Louisville is also appreciated.

\section{References}

Assmann, A., \& Reinhardt, H. W. (2014). Tensile creep and shrinkage of SAP modified concrete. Cement and Concrete Research, 58, 179-185.

Bentz, D. P., \& Snyder, K. A. (1999). Protected paste volume in concrete: Extension to internal curing using saturated lightweight fine aggregate. Cement and concrete research, 29(11), 1863-1867.

Gugliemelli, L. A., Doane, W. M., Russell, C. R., Swanson, C. L., Arcand, C. G., \& Vullo, W. J. (1972). 2264201. Starch-cerium (IV) complexes in aqueous media: formation, isolation, and stability. Journal of polymer science: Part B: Polymer letters, 10(6), 415-421.

Igarashi, S. I., \& Watanabe, A. (2006). Experimental study on prevention of autogenous deformation by internal curing using super-absorbent polymer particles. In International RILEM conference on volume changes of hardening concrete: testing and mitigation (pp. 77-86). RILEM Publications SARL.

Jensen, O. M., \& Hansen, P. F. (2001). Water-entrained cement-based materials: I. Principles and theoretical background. Cement and concrete research, 31(4), 647-654.

Jensen, O. M., \& Hansen, P. F. (2002). Water-entrained cement-based materials: II. Experimental observations. Cement and Concrete Research, 32(6), 973-978.

Lipsa, R., Tudorachi, N., Vasile, C., Chiriac, A., \& Grigoras, A. (2013). Novel environmentally friendly copolymers carboxymethyl starch grafted poly (lactic acid). Journal of Polymers and the Environment, 21(2), 461-471.

Liu R. J. (2003). Preparation and Properties of Organic-inorganic Multiple Concrete Internal Curing Materials. Ph.D. Thesis. Wuhan University of Technology, Wuhan.

Lura, P., Wyrzykowski, M., Tang, C., \& Lehmann, E. (2014). Internal curing with lightweight aggregate produced from biomass-derived waste. Cement and concrete research, 59, 24-33.

Lutfor, M. R., Rahman, M. Z. A., Sidik, S., Mansor, A., Haron, J., \& Yunus, W. W. (2001). Kinetics of graft copolymerization of acrylonitrile onto sago starch using free radicals initiated by ceric ammonium nitrate. Designed Monomers and Polymers, 4(3), 252-259.

Mindess, S., Young, J. F., \& Darwin, D. (2003). Concrete, $2^{\text {nd }}$ Ed. Upper Saddle River: New York, pp.T22

Moad G., Solomon D. H. (2006). The Chemistry of Radical Polymerization, 2nd ed. Elsevier, Amsterdam.

Philleo, R. (1991). Materials science of concrete II. American Ceramic Society, Westerville, OH, USA, 1-8.

Rahman, L., Silong, S., Zin, W. M., Rahman, M. Z. A., Ahmad, M., \& Haron, J. (2000). Graft copolymerization of methyl acrylate onto sago starch using ceric ammonium nitrate as an initiator. Journal of applied polymer science, 76(4), 516-523.

Ren, H., Niu, Z., Wang, J., \& Ning, J. (2012). Comparison of Traditional Methods and Microwave Irradiation Method About Amylum/Acrylic Acid/Acrylamide Polymerization. Edited by Ailton De Souza Gomes, 87.

Schröfl, C., Mechtcherine, V., \& Gorges, M. (2012). Relation between the molecular structure and the efficiency of superabsorbent polymers (SAP) as concrete admixture to mitigate autogenous shrinkage. Cement and concrete research, 42(6), 865-873. 
Tong Q. Y., Zhang G. W., (2005). Graft polymerization of acrylonitrile and amps onto starch initiated by $\left[\mathrm{Mn}\left(\mathrm{H}_{2} \mathrm{P}_{2} \mathrm{O}_{7}\right)_{3}\right]^{3-}$. Polym. Mater. Sci. Eng. 21(1), 106-109

Wang, B. J. (2003) Study on the Synthesis and Kinetics of Starch-based Graft Copolymer. Ph.D. Thesis, Nanjing University of Technology, Nanjing.

Weber, S., \& Reinhardt, H. W. (1997). A new generation of high performance concrete: concrete with autogenous curing. Advanced Cement Based Materials, 6(2), 59-68.

Yang, B., Zhao, Y. L., Yang, W. M., \& Wang, J. Y. (2006). Kinetics Equation of Radiative Graft Copolymerization of Starch and Acrylamide. Yunnan Chemical Technology, 33(3), 8-10.

Yu, F. Q., Yao, S., \& Liu, Y. (1999). Kinetics of graft copolymerization of an onto corn starch under UVlight. Polym. Mater. Sci. Eng., 15(5), 62-65.

Zhang Y. P., (2001). Manufacture and application of modified starch. Chemical Industry Press, Beijing. 